\title{
Modification of domain-wall propagation in Co nanowires via $\mathrm{Ga}^{+}$ irradiation
}

L. Serrano-Ramón ${ }^{1,2}$, A. Fernández-Pacheco ${ }^{3}$, M. R. Ibarra ${ }^{2,4}$,D. Petit ${ }^{3}$, R.P. Cowburn ${ }^{3}$, T. Tyliszczak ${ }^{5}$ and J. M. De Teresa ${ }^{1,2,4, *}$

${ }^{1}$ Instituto de Ciencia de Materiales de Aragón, Facultad de Ciencias, Universidad de ZaragozaCSIC, E-50009 Zaragoza, Spain

${ }^{2}$ Departamento de Física de la Materia Condensada, Universidad de Zaragoza, E-50009 Zaragoza, Spain

${ }^{3}$ TFM Group, Cavendish Laboratory, University of Cambridge,JJ Thomson Avenue, CB3 0HE, Cambridge,United Kingdom

${ }^{4}$ Laboratorio de Microscopías Avanzadas (LMA), Instituto de Nanociencia de Aragón (INA), Universidad de Zaragoza, E-50018 Zaragoza,Spain

${ }^{5}$ Advanced Light Source, Lawrence Berkeley National Laboratory, USA

*E-mail: deteresa@unizar.es

Section: Solid State and Materials

Suggested Editor: Francesca Casoli

\begin{abstract}
The propagation of domain walls in polycrystalline Co nanowiresgrown by focused-electronbeam-induced depositionis explored. We have found that $\mathrm{Ga}^{+}$irradiation via focused ion beam is a suitable method to modify the propagation field of domain walls in magnetic conduits.
\end{abstract}


Magneto-optical Kerr effect measurements show that global $\mathrm{Ga}^{+}$irradiation of the nanowires increases the domain-wall propagation field. Additionally, wehave observedby means of scanning transmission X-ray microscopy that it is possible to produce substantial domain-wall pinning via local $\mathrm{Ga}^{+}$irradiation of a narrow region of the nanowire.In both cases, $\mathrm{Ga}^{+}$doses of the order of $10^{16}$ ions $/ \mathrm{cm}^{2}$ are required to produce such effects. These results pave the way for the controlled manipulation of domainwalls in Co nanowires via $\mathrm{Ga}^{+}$irradiation. 


\section{Introduction}

Tailored magnetic nanostructures are currently at the core of several existing applications in magnetic storage and sensing [1-3]. Further applications of magnetic nanostructures are being intensively explored such as those related to the manipulation of domain walls. For instance, proposals have been put forward for their use in magnetic logic devices [4], in the racetrack memory [5] or for biotechnology applications [6]. All these devices rely on magnetic domain wall conduits, where the propagation field is lower than the nucleation field, making possible the motion and manipulation of domain walls by applying external magnetic fields [7]. Additionally, domain walls can be propagated in wires by means of electrical current pulses [8-10].

Some methods have been explored to allow the manipulation of domain walls in such magnetic domain wall conduits. Artificial notches [11], artificial arms [12], localized stray fields [13] or electric fields $[14,15]$ have been reported to modify the propagation of domain walls, thus being potential elements in future magnetic circuits. Ion irradiation produces substantial changes in the magnetic anisotropy of magnetic materials, as extensively shown in materials with perpendicular magnetic anisotropy [16-22] and to a lesser extent in materials with in-plane magnetization [23-25]. Thus, ion irradiation is a promising route to tailor the propagation of domain walls in magnetic domain wall conduits. $\mathrm{Ga}^{+}$FIB irradiation has been recently proposed as a route to create shift registers based on magnetic domain wall ratchets with perpendicular anisotropy [26].

In the present article, we report a detailed study of the use of $\mathrm{Ga}^{+}$irradiation in order to modify the propagation field of domain walls in Co nanowires grown by focused-electronbeam-induced deposition (FEBID). For the growth of the nanostructures presented hereafter, $\mathrm{Co}_{2}(\mathrm{CO})_{8}$ is used as the gas precursor. A Scanning Electron Microscope activates the molecule dissociation, forminga nanopatterned Co nanostructure with the desired shapein a single lithography step [27].In the following, we present two different routes to explore the modification of domain wall propagation fields. First, L-shaped nanowires are globally irradiated and the modification of the domain wall propagation field is measured by means of 
Magneto-Optical Kerr Effect (MOKE) [28]. These results allow us to estimate the ion doses required for pinning domain walls in this material.In the second route, a narrow central area of a long nanowire is irradiated, and the pinning of a domain wall at that area is imaged by means of Scanning Transmission X-ray Microscopy (STXM) [29].

\section{Experimental Section}

Sample growth and subsequent $\mathrm{Ga}^{+}$irradiation(without breaking the vacuum) were performed inside a commercial Helios 600 Dual Beam system, which combines a Scanning Electron Microscope (SEM) and a Focused Ion Beam (FIB). The base pressure in the chamber is $10^{-6} \mathrm{mbar}$. For the growth of Co nanostructures, a gas-injection system heated to $27^{\circ} \mathrm{C}$ allows the introduction of the $\mathrm{Co}_{2}(\mathrm{CO})_{8}$ precursorin the proximity of the working area. The nozzle tip through which the gas is released is located $50 \mu \mathrm{m}$ above the substrate and $150 \mu \mathrm{m}$ off the central working area. The deposit grows on the areas scanned by the SEM, this technique being known asFEBID [27]. It was previously shown that functional polycrystalline Co nanostructures can be grown by this technique [30], with Co content above 95\% [31] and with a resolution of $30 \mathrm{~nm}$ [32, 33]. The Co nanostructures shown in the present work have been grown using the following parameters: process pressure $=1.9 \cdot 10^{-5}$ mbar, beam voltage $=10 \mathrm{kV}$, beam current $=2.7 \mathrm{nA}$.

Hereafter, the two different types of samples studied are described.In the first type,sketched in figure 1 and named "Route 1", Si substrates are used. The first step consists of the growth of L-shaped Co wires ending on both sides with a pointed shapeto avoid nucleation of domain walls at the ends. This "L" shape is suitable for the systematic investigation of propagation fields in nanowires [34, 35]. Three batches of samples were prepared, corresponding to three different nanowire widths.As measured by AFM, the width of samples in batches 1, 2 and 3 before ion irradiation are respectively,on average, $310 \mathrm{~nm}, 370 \mathrm{~nm}$ and 490 $\mathrm{nm}$. The thicknesses measured by atomic force microscopy (AFM) are respectively $72 \mathrm{~nm}, 70$ $\mathrm{nm}$ and $84 \mathrm{~nm}$ for batch 1, 2 and 3.In a second step, the nanowire is globally irradiated by $\mathrm{Ga}^{+}$ FIB. The beam voltage and current used for the irradiation are respectively $30 \mathrm{kV}$ and $48 \mathrm{pA}$ (93 
pA for high ion irradiation doses). Each batch contains 18 samples, created with varying $\mathrm{Ga}^{+}$ dose,up to $1.25 \times 10^{17}$ ions $/ \mathrm{cm}^{2}$. The propagation field of domain walls in these samples was subsequently investigated using MOKE. For that, a high magnetic field is applied at $+45^{\circ}$ degrees (see figure 1), which saturates the magnetization of the two arms, creating a domain wall at the corner. Afterwards, the applied magnetic field is set to zero and, subsequently, only a horizontal negative magnetic field is applied (which is parallel to the long arm), moving the domain wall to the right side at the propagation field. The switching of the magnetization in the long arm of the structure is then detected as the laser beam is focused on it [35]. Similarly, the complementary magnetic cycle is built by applying the saturating magnetic field at $-45^{\circ}$, setting the magnetic field to zero and applying a horizontal positive magnetic field.

For STXM experiments, we used a different geometry, since only unidirectional magnetic fields can be applied. The name given in figure 1 to this approach is "Route 2". 50 nm-thick $\mathrm{Si}_{3} \mathrm{~N}_{4}$ membranes were used as substrates to allow sufficient $\mathrm{x}$-ray transmission. The cobalt thickness on the $\mathrm{Si}_{3} \mathrm{~N}_{4}$ membranes is roughly $45 \mathrm{~nm}$. In this approach, the first step consists of the growth of long Co wires (length $=15 \mu \mathrm{m}$, width $=250 \mathrm{~nm}$ ) with a pointed end on the left and an oval shape on the right side to facilitate the nucleation of a domain wall and its subsequent injection in the nanowire [36]. In a second step, a narrow area(width $=250 \mathrm{~nm}$ )of the central part of the nanowire is irradiated by $\mathrm{Ga}^{+}$FIB. The beam voltage and current used in these experiments are respectively $30 \mathrm{kV}$ and 1.5pA (9.8 pA for high irradiation doses). Several samples were prepared with $\mathrm{Ga}^{+}$doses ranging from $1.87 \times 10^{13}$ ions $/ \mathrm{cm}^{2}$ up to5.97x $10^{16}$ ions $/ \mathrm{cm}^{2}$. The magnetization reversal in these samples was subsequently imaged by STXM. These measurements were carried out at beamline 11.0.2 of the Advanced Light Source, Lawrence Berkeley Laboratory [29]. Soft $\mathrm{x}$-rays are focused on the sample with a spot of $\approx 30 \mathrm{~nm}$. The $\mathrm{x}-$ ray beam is scanned on the sample and the transmitted signal is measured. The $\mathrm{L}_{3}$ absorption edge of cobalt, at $778 \mathrm{eV}$, is used in the experiments. The samples are tilted $30^{\circ}$ with respect to theplane perpendicular to the $\mathrm{x}$-ray beam in order to be sensitive to the in-plane magnetization [37]. 
The magnetization reversal is imaged due to the magnetic contrast arising from the $\mathrm{x}$ ray magnetic circular dichroic contribution to the total signal. The experiments are performed at a fixed polarization and varying the external magnetic field. The magnetic image at a given field is obtained by digitally subtractingthe image at magnetic saturation. Thus, the grey scale corresponds to the changes in the $\mathrm{x}$ component of the magnetization. Black (white) contrast means that the magnetization is negatively (positively) saturated.

\section{Results}

\subsection{Global $\mathrm{Ga}^{+}$irradiation of $\mathrm{L}$-shaped Co nanowires}

SEM images of the L-shaped nanowires, before and after global $\mathrm{Ga}^{+}$FIB irradiation, are shown in figures2(a) and 2(b) respectively. The morphology changes produced by $\mathrm{Ga}^{+}$ irradiation are clearly noticeablein this particular nanowire due to the high irradiation dose of $3.11 \times 10^{16}$ ions $/ \mathrm{cm}^{2} . \mathrm{Ga}^{+}$irradiation is known to produce changes such as material etching, Ga implantation, microstructural modifications, etc. A detailed investigation of these changes will be the subject of a future publication as it requires specific transmission electron microscopy studies beyond the scope of the present manuscript [38].

The domain wall propagation field of the nanowires has been systematically studied by MOKE for the three batches, as described above. As an example, we show in figure 3 individual MOKE hysteresis loops corresponding to batch 1 . At a low irradiation dose, $0.147 \times 10^{16}$ ions $/ \mathrm{cm}^{2}$, see figure 3(b), it can be noticed that the propagation field as well as the absolute value of the Kerr signal remain similar to the non-irradiated nanowire, shown in figure 3(a). This indicates that such a low dose does not significantly affect the sample morphology or the magnetic properties of the nanowire. The situation is different for a nanowire with anion dose ten times higher, $1.56 \times 10^{16}$ ions $/ \mathrm{cm}^{2}$. As shown in figure 3(c), in this case the propagation field has increased by about $50 \%$ and the absolute value of the Kerr signal has decreased by a factor of three. These facts point towards significant changes in the morphology and the magnetic properties of nanowires with $\mathrm{Ga}^{+}$irradiations for that range of doses. The same trend is readily 
noticeable for a nanowire with an irradiation dose of $6.23 \times 10^{16} \mathrm{ions} / \mathrm{cm}^{2}$, shown in figure 3(d), where the propagation field has increased by a factor of three with respect to the original nonirradiated nanowire. Also, the absolute value of the Kerr signal is only one sixth of the original value. The magnitude of the Kerr signal is not a direct measurement of the magnetization of the material probed [39]. However, the decrease observed upon FIB irradiation is a good indication of the expected decrease of the magnetization with $\mathrm{Ga}^{+}$bombardment.

The set of results obtained for the three batches studied is summarized in figure 4. In all cases, and beyond the expected statistical distribution of values, we observe a clear increase of the propagation field with $\mathrm{Ga}^{+}$dose. The figure shows that a globalirradiation doseof $4 \times 10^{16}$ ions/ $\mathrm{cm}^{2}$ typically produces an increase in the domain wall propagation field of about $100 \%$.We anticipate that $\mathrm{Ga}^{+}$irradiation doses above that value arerequired to produce domain wall pinning in nanowires when a local $\mathrm{Ga}^{+}$irradiation is performed, as shown below.

\subsection{Local $\mathrm{Ga}^{+}$irradiation of Co nanowires}

This approach, assketched in figure 1, consists on the $\mathrm{Ga}^{+}$irradiation of a narrow central area of the nanowire, aiming at the effective pinning of a domain wall, as it propagates from the pad to the other extremity of the wire. As shown in section 3.1, a Ga ${ }^{+}$irradiated area presents a higher domain wall propagation field. Thus, a domain wall moving from the oval pad located on the right towards the left side is likely to become pinned at the irradiated central part of the nanowire. Previously, it was demonstrated that FIB irradiated areas in Permalloy films become effective pinning places for domain walls [23]. The mechanisms behind this effect can be manifold and depend on several parameters but morphological changes produced by the irradiation were found to be relevant [23].

Several devices with varying irradiation doses were imaged by STXM. For dosesbelow $4 \times 10^{16}$ ions $/ \mathrm{cm}^{2}$, no effective pinning at the irradiated area was observed. In that case, the domain wall is injected from the oval pad into the nanowire and travels immediately to the left end. However, for doses above $4 \times 10^{16}$ ions $/ \mathrm{cm}^{2}$, we observed that the domain wall 
become effectively pinned at the position where the FIB process was applied, as shown in figure 5. In that figure, one can observe that at +48 Oe the oval pad has reversed its magnetization except in the topmost part, thus being ready to inject a domain wall into the nanowire. At +50 Oe, a domain wall is injected in the nanowire and becomes pinned at the irradiated area. At +64 Oe the full nanowire magnetization is reversed, most likely due to the domain wall depinning from the area where it was trapped. Renucleation from the left side of the wire cannot discarded but is unlikely, as experiments in similar wires without pad show higher nucleation fields.

In figure 6 another interesting sample is shown, where the irradiation dose is $5.97 \times 10^{16}$ ions $/ \mathrm{cm}^{2}$. In this case, a domain wall is injected from the oval pad at +10 Oe. The domain wall getsfirst pinned at a random defect located before reaching the irradiated region. The defect is clearly visible in the STXM images. The domain wall remains pinned at this position before a magnetic field of +52 Oe is applied, when it becomes depinned and travels to the left, getting pinned at the artificial trap created by the FIB. It remains pinned there until a magnetic field of +56 Oe is applied, where the full length of the nanowire has reversed its magnetization.

Summarizing the results obtained in these two samples, one can conclude that local $\mathrm{Ga}^{+}$ FIB irradiation can be an effective way to create artificial domain wall traps in Co nanowires grown by FEBID. The threshold dose to produce such pinning is about $4 \times 10^{16}$ ions $/ \mathrm{cm}^{2}$.

\section{Discussion}

Ion irradiation is becoming a powerful technique to modify the magnetic properties of nanostructures, sometimes creating novel and exciting devices [26]. Present technologies such $\mathrm{asGa}^{+}$FIB irradiation used here allow local ion irradiation at the level of a few nanometers, giving rise to specific magnetic nano-patternings with high resolution and potential integration for applications in magnetic storage and sensing [17, 27]. The use of focused ion irradiation to create a pinning landscape and eventually manipulate domain walls in magnetic conduits is starting to be considered as an alternative route to other more conventional approaches. On the other hand, the FEBID technique is a candidate to become the ultimate-resolution 
nanolithography technique. Indeed, $3 \mathrm{~nm}$ dots have been grown inside a scanning electron microscope [40]. We have recently shown that it is possible to grow functional Co-based ferromagnetic nanowires using FEBID [35, 41], reaching so far $30 \mathrm{~nm}$ resolution [32].

To our knowledge, the work presented here is the first attempt to combine inside the same process chamber the growth of ferromagnetic conduits and subsequent ion irradiation. In just two steps it is possible to create ion-irradiated ferromagnetic nanostructures without the difficulty of using resist and keeping high-vacuum conditions all the time. As a consequence, net advantages of this approach are the short total process time and the minimization of any potential contamination.

Hereafter, we discuss the possible origin behind thedomain wall pinning produced by local irradiation as well as the increase of propagation fields observed by global irradiation.The system studied here, Co nanowires with in-plane magnetization, is substantially different from most of previous studies, which focused on films or wireswith perpendicular magnetic anisotropy (PMA). In the PMA case, low $\mathrm{Ga}^{+}$irradiation doses, $\approx 10^{13}$ ions $/ \mathrm{cm}^{2}$, promote interface mixing, resulting in a substantial decrease of PMA [16]. However, for in-plane magnetized materials such as Permalloy films, previous studies reported thatthe dose required for domain wall pinning is substantially higher, $\approx 10^{16} \mathrm{ions} / \mathrm{cm}^{2}[23]$. The experiments performed here show that doses in the same range are necessary to pin domain walls in polycrystalline Co nanowires grown by FEBID.

For these high dose values, the high energetic ions are expected to produce substantial modification in the material such as etching of the exposed area, Ga implantation at (and around) the exposed area, or creation of defects, which will likely diminish the sample magnetization. Ozkaya and co-workers have also reported changes in the sample grain size and the lattice parameters [23], whereas other authors report amorphization effects [42, 43].All these factors will alter the energy landscape sensed by a moving domain wall, with all terms, anisotropy, magnetostatic, magnetoelastic, and exchange energy being modified. In order to discern which effect is predominant, it is necessary to know in detail the exact morphological changes produced in these Co nanowires when irradiated, which is currently in progress and 
will be published elsewhere [38].The first results indicate that the global Ga irradiation (route 1) produces the following effects in our samples. First, etching of the halo around the deposits as well as the creation of a top-surface shell with larger cobalt grain size are observed. As the Ga irradiation further proceeds, the volume and thickness of the wires is significantly decreased whereas the roughness increases. These effects correlate with the following features observedwith increasing Ga irradiation: the decrease of the absolute value of the MOKE signal and the increase in the domain-wall propagation field. The morphological changes induced by the Ga irradiation will cause domain-wall pinning, perturbing the domain wall propagation, which will require higher magnetic fields.

\section{Summary and conclusions}

Summarizing, we have grown cobalt domain wall conduits by focused-electron-beaminduced deposition (FEBID) and subsequentlyirradiated them using $\mathrm{Ga}^{+}$FIB irradiation. By performing a systematic study of how the propagation fields of domain walls change with the ion dose, we have found that doses in the range of $10^{16}$ ions $/ \mathrm{cm}^{2}$ are required for an effective pinning of domain walls. This effect has been observed in both, L-shaped Co nanowires under a global $\mathrm{Ga}^{+}$FIB irradiation, as well as in locally irradiated linear Co nanowires, where the trapping of domain walls was directly imaged. The conditions and strategies for future engineering of ion-irradiated magnetic conduits based on Co nanowires grown by FEBID have thus been established.

\section{Acknowledgements}

We acknowledge Dr. R. Córdoba for experimental help, as well as Dr. M. Mansell for critically reading the manuscript.Financial support by Spanish Ministry of Science (through project MAT2011-27553-C02, including FEDER funding), CSIC through the I-LINK-026 projectand the Aragon Regional Government is acknowledged.A.F.-P. acknowledges support by 
a Marie Curie IEF within the 7th European Community Framework Programme No. 251698, 3DMAGNANOW. 


\section{References}

1.C.Chappert, A.Fert and F.N.Van Dau, Nature Mat.6, 813 (2007)

2.I.R. McFadyen, E.E. Fullerton and M.J. Carey, MRS bulletin 31, 379 (2006)

3. D.L. Graham, H.A. Ferreira, P.P. Freitas, Trends Biotechnol. 22,428 (2004)

4.D.A. Alwood, G. Xiong, C.C. Faulkner, D. Atkinson, D. Petit, R.P. Cowburn, Science 309, $1688(2005)$

5.S.S.P.Parkin, M.Hayashi and L.Thomas,Science320, 190 (2008)

6.M.Donolato et al., Adv. Mater. 22, 2706(2010)

7. R.P. Cowburn, D.A. Allwood, G. Xiong, M.D. Cook, J. Appl. Phys.91, 6949(2002)

8.A. Yamaguchi, T. Ono, S. Nasu, K. Miyake, K. Mibu, T. Shinjo, Phys. Rev. Lett. 92, 077205 (2004)

9.G. Malinowski, O. Boulle and M. Kläui, J. Phys. D: Appl. Phys. 44,384005 (2011)

10. A. Brataas, A.D. Kent and H. Ohno, Nature Materials 11,372 (2012)

11. M. Kläui, C.A.F. Vaz, J.A.C. Bland, W. Wernsdorfer, G. Faini, E. Cambril, J. Appl. Phys. 93,7885 (2003)

12. E.R. Lewis et al., Nature Materials 9, 980 (2010)

13. L. O`Brien et al., Phys. Rev. Lett. 106, 087204 (2011)

14.A.J. Schellekens, A. Van den Brink, J.H. Franken, H.J.M. Swagten, and B. Koopmans, Nature Communications 3,847(2012)

15. D. Chiba et al., Nature Communications 3, 888(2012)

16. J.H. Franken, M. Hoeijmakers, R. Lavrijsen and H.J.M. Swagten, J. Phys.: Condens. Matter 24, $024216(2012)$

17. C. Chappert et al., Science 280, 1919 (1998)

18. T. Gerhardt, A. Drews, G. Meier, J. Phys.: Condens. Matter 24, 024208 (2012)

19. J. Fassbender, J. McCord, J. Magn. Magn.Mater.320, 579 (2008)

20. D. Weller, J. E. E. Baglin, A.J. Kellock, K.A. Hannibal, M.F. Toney, J. Appl. Phys.87, 5768 (2000). 
21. C. Vieu, J. Gierak, H. Launois, T. Aign, P. Meyer, J. P. Jamet, and J. Ferre, C. Chappert, T. Devolder, V. Mathet and H. Bernas, Journal of Applied Physics91, 3103 (2002)

22. M. Cormier et al., J. Phys D: Appl. Phys.44, 215002 (2011)

23 D. Ozkaya, R.M. Langford, W.L. Chan, A.K. Petford-Long, J. Appl. Phys.91, 9937 (2002)

24. J. Barzola-Quiquia, S.Dusari, G.Bridoux, F. Bern, A.Molle and P.Esquinazi, Nanotechnology21, 145306 (2010)

25. T.T. Suzuki, H. Kuwahara, Y. Yamauch, Surface Science 605, 1197 (2011)

26J.H. Franken, H.J.M. Swagten, B. Koopmans, Nature Nanotechnology 7, 499 (2012)

27.P.E. Russell, I. Utke, S. Moshkalev (Editors), Nanofabrication using focused ion and electron beams: principles and applications, Oxford University Press, NY, USA, ISBN: 9780199734214 (2012)

28.D.A.Alwood, G.Xiong, M.D. Cooke and R.P.Cowburn,J. Phys D: Appl. Phys. 36, 2175 (2003)

29.A.L.D. Kilcoyne et al., Journal of Synchrotron Radiation 10, 125 (2003)

30. I. Utke, P. Hoffmann, R. Berger and L. Scandella, Appl. Phys. Lett. 80,4792(2002)

31.A. Fernández-Pacheco, J.M. De Teresa, R. Córdoba and M.R. Ibarra,J. Phys. D: Appl. Phys. 42,055005(2009)

32. L. Serrano-Ramón, R. Córdoba, L. A. Rodríguez, C. Magén, E. Snoeck, C. Gatel, I. Serrano, M. R. Ibarra, J. M. De Teresa, ACSnano5, 7781 (2011)

33. E. Nikulina, O. Idigoras, P. Vavassori, A. Chuvilin, A. Berger, Appl. Phys. Lett. 100, 142401(2012)

34. D. A. Allwood, N. Vernier, Gang Xiong, M. D. Cooke, D. Atkinson, C. C. Faulkner, and R. P. Cowburn, Appl. Phys. Lett. 81, 4005 (2002)

35. A. Fernández-Pacheco, J.M. De Teresa, R. Córdoba and M.R. Ibarra, D. Petit, D.E. Read, L.O’ Brien, E.R. Lewis, H.T. Zeng and R.P. Cowburn, Appl. Phys. Lett. 94, 192509 (2009)

36. M.-Y. Im, L. Bocklage, P. Fischer, and G. Meier, Phys. Rev. Lett. 102, 147204 (2009)

37. A. Fernández-Pacheco et al. Nanotechnology 23, 105703 (2012)

38. L. Serrano-Ramón et al., to be published 
39. Z.Q. Qiu and S.D. Bader, J. Magn. Magn.Mater.200,664 (1999)

40. L. van Kouwen, A. Botman, C.W. Hagen, Nano Lett.9, 2149 (2009)

41. A. Fernández-Pacheco, J.M. De Teresa, A. Szkudlarek, R. Córdoba and M.R. Ibarra, D.

Petit, L. O’Brien, H.T. Zeng, E.R. Lewis, D.E. Read and R.P. Cowburn, Nanotechnology 20,475704(2009)

42.D. McGrouther, J.N. Chapman, Appl. Phys. Lett. 87, 022507 (2005)

43.E. Arac, D. M. Burn, D. S. Eastwood, T.P.A. Hase, D. Atkinson, J. Appl. Phys. 111, 044324 (2012) 


\section{Figure captions:}

Figure 1. Sketches of the two different routes followed for the investigation of the influence of $\mathrm{Ga}^{+}$FIB irradiation on the domain wall propagation in Co nanowires grown by FEBID. (a) Route 1: global $\mathrm{Ga}^{+}$FIB irradiation.(b) Route 2: local $\mathrm{Ga}^{+}$FIB irradiation. See text for detailed explanations.

Figure 2. SEM images of an L-shaped nanowire before and after global $\mathrm{Ga}^{+}$FIB irradiation, with a dose of $6.24 \times 10^{16}$ ions $/ \mathrm{cm}^{2}$.

Figure 3. MOKE hysteresis loops of L-shaped nanowires (batch 1) under increasing irradiation dose.

Figure 4. Domain-wall propagation field as a function of irradiation dose for three different batches of L-shaped nanowires.

Figure 5. STXM sequence of images of the magnetization reversal in one of the devices with local $\mathrm{Ga}^{+}$FIB irradiation (irradiation dose of $4.48 \times 10^{16} \mathrm{ions} / \mathrm{cm}^{2}$ ).

Figure 6. STXM sequence of images of the magnetization reversal in one of the devices with local $\mathrm{Ga}^{+}$FIB irradiation (irradiation dose of $5.97 \times 10^{16}$ ions $/ \mathrm{cm}^{2}$ ). A random defect is able to pin the domain wall before reaching the central area of the nanowire. 
FIGURE 1

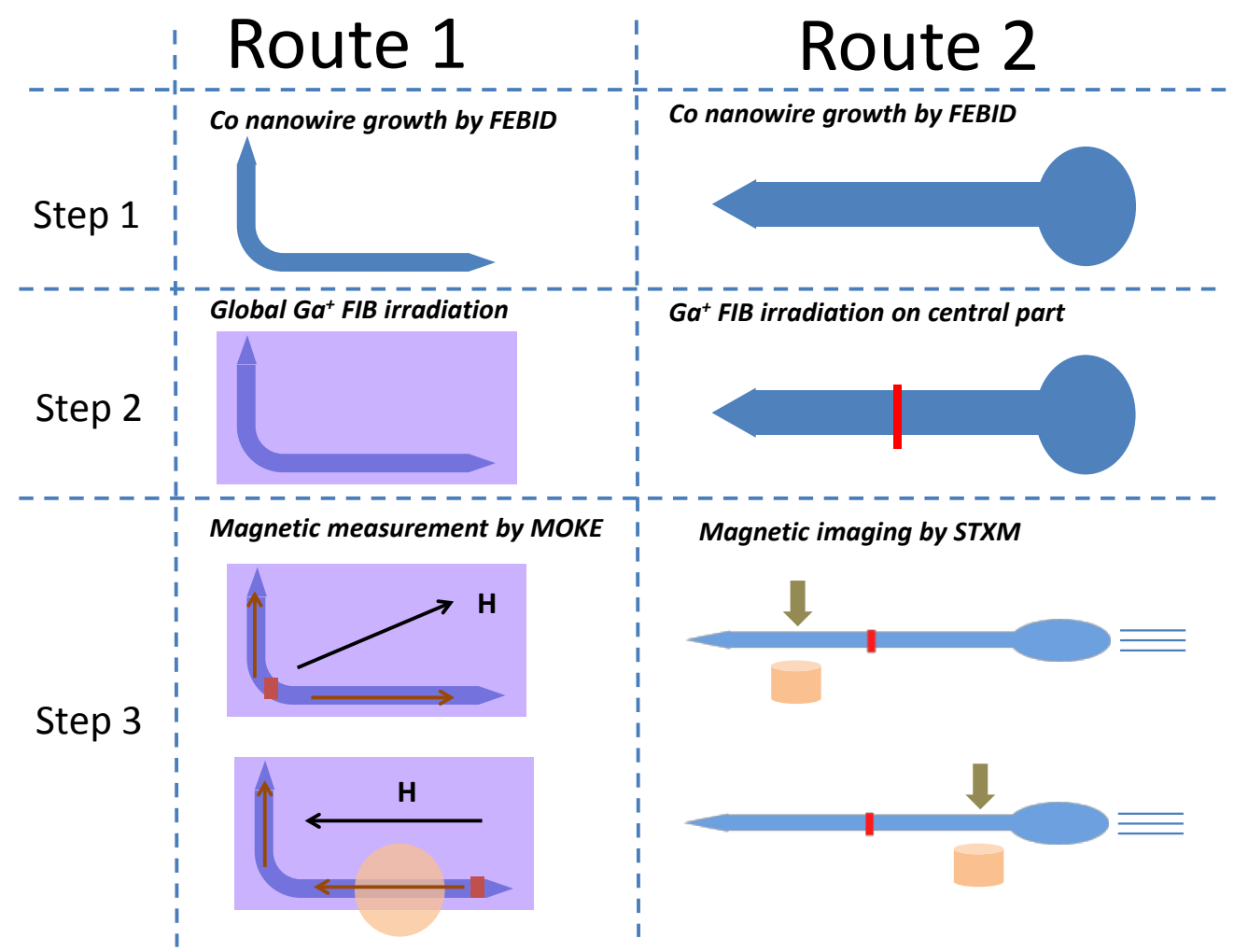


FIGURE 2

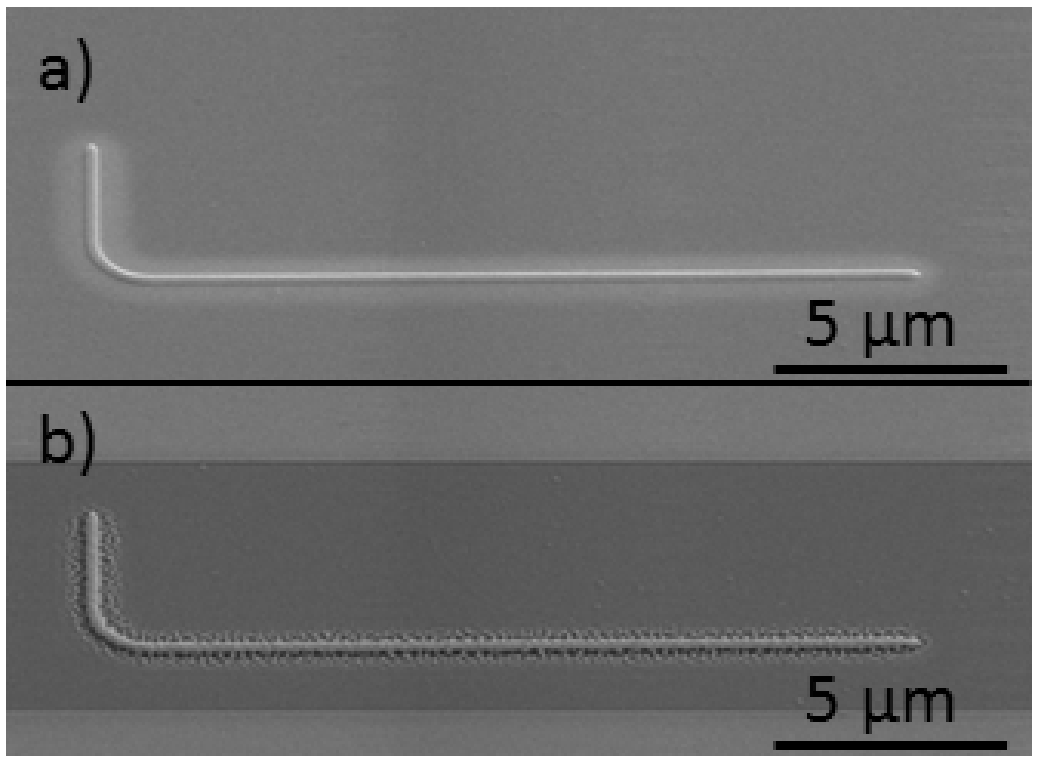


FIGURE 3
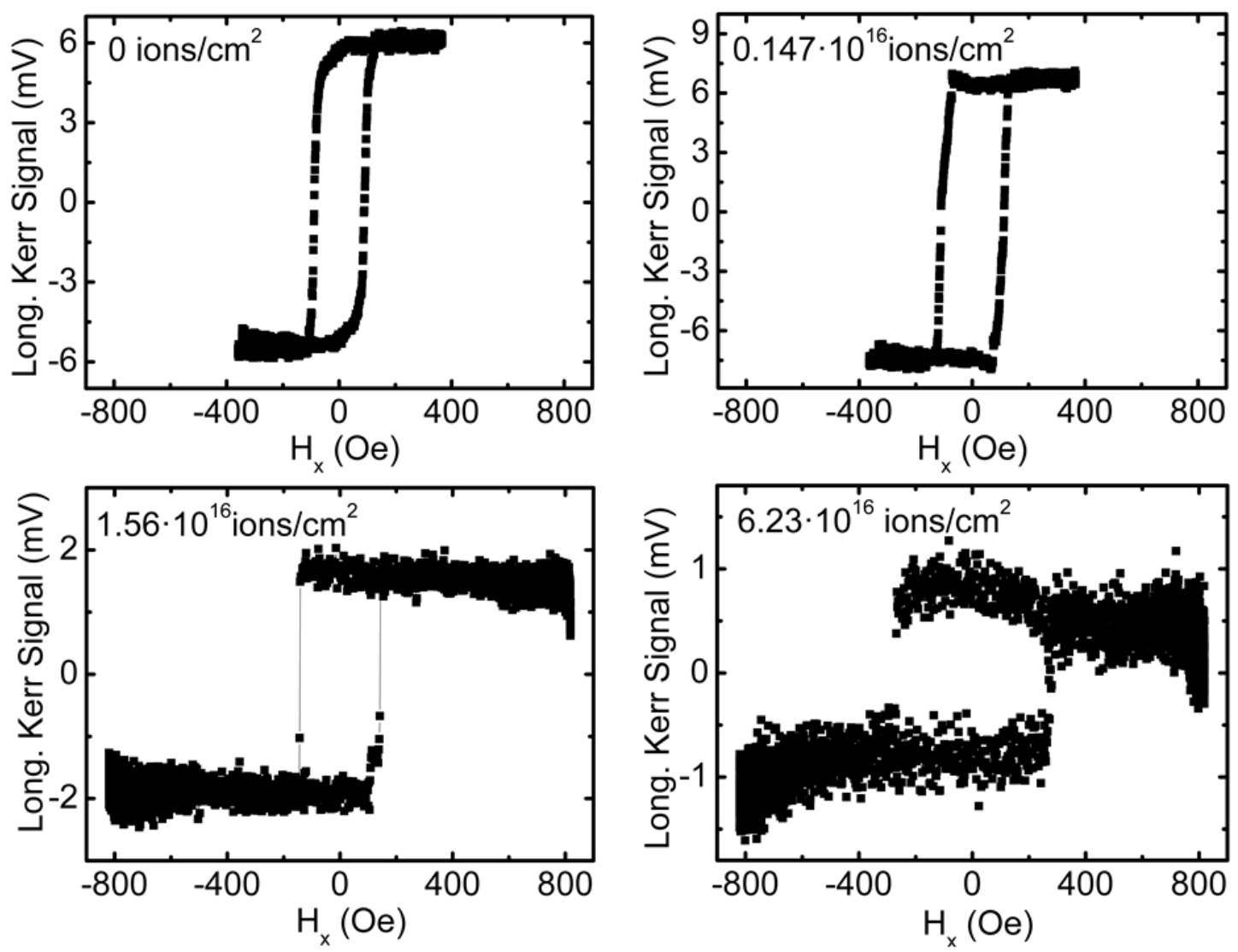
FIGURE 4

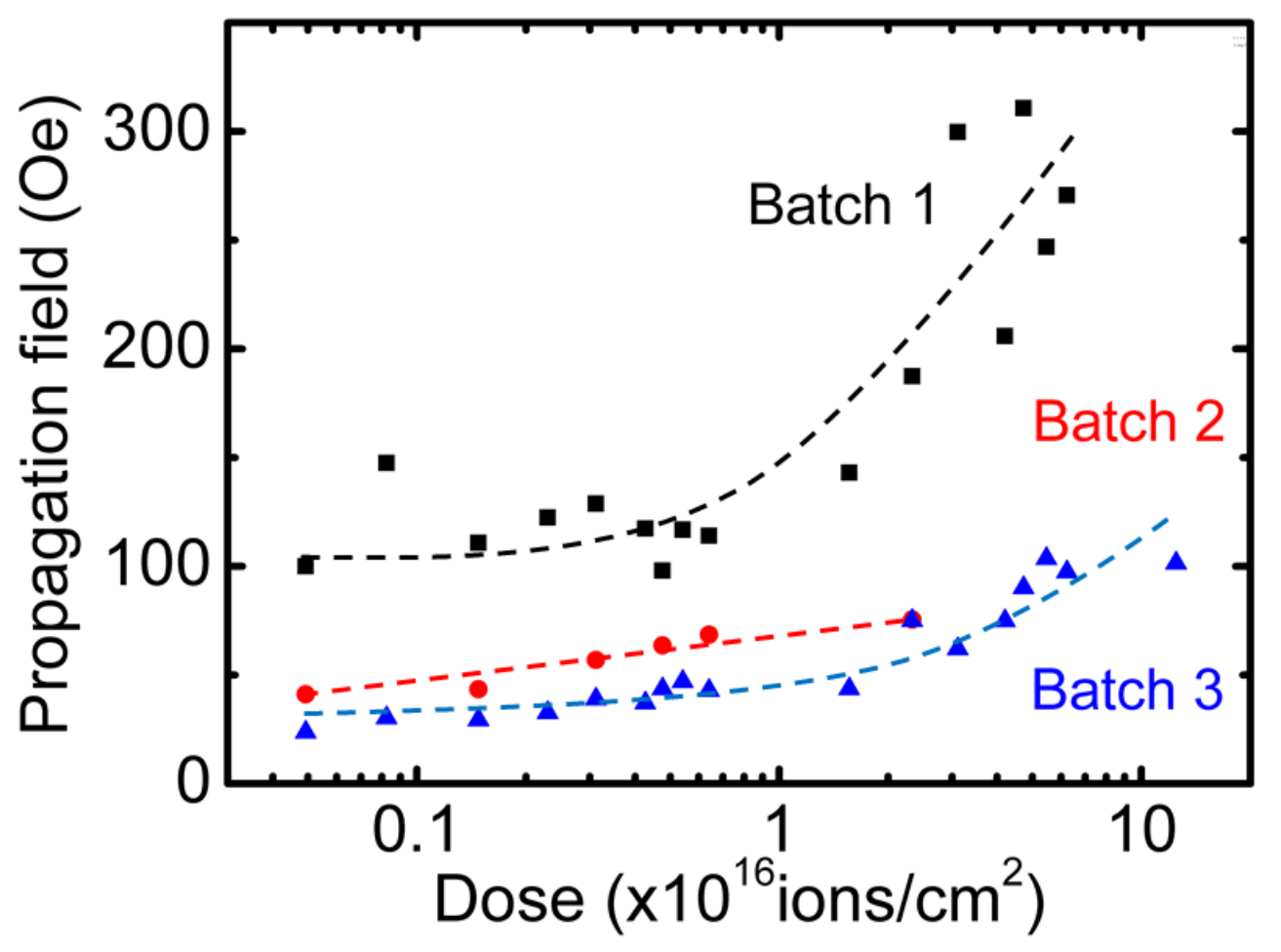


FIGURE 5
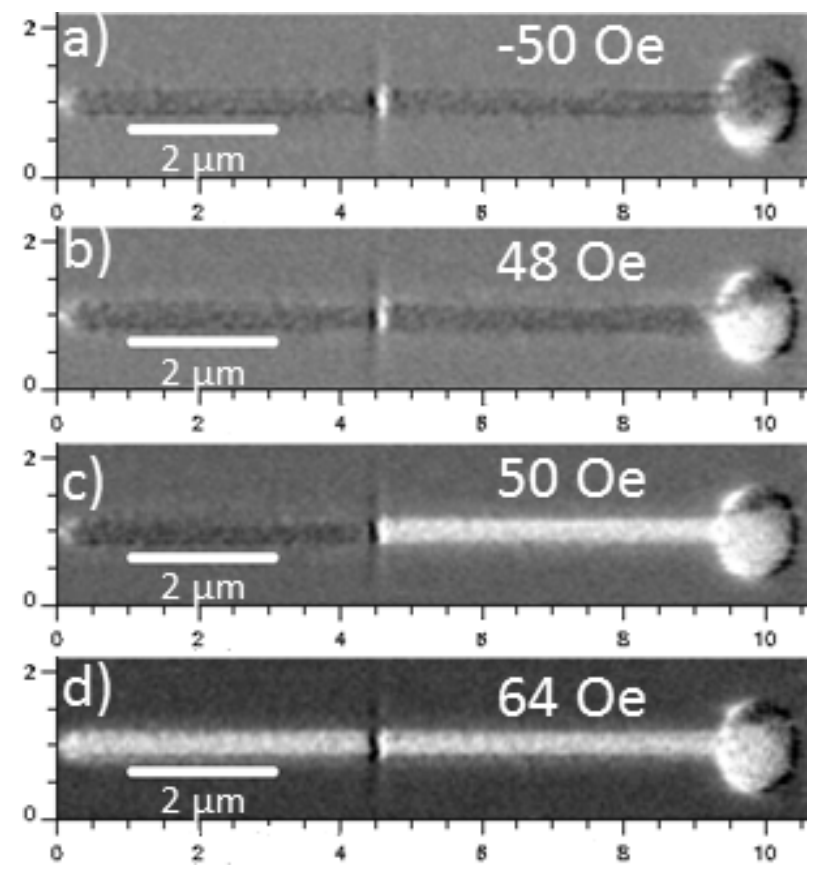
FIGURE 6
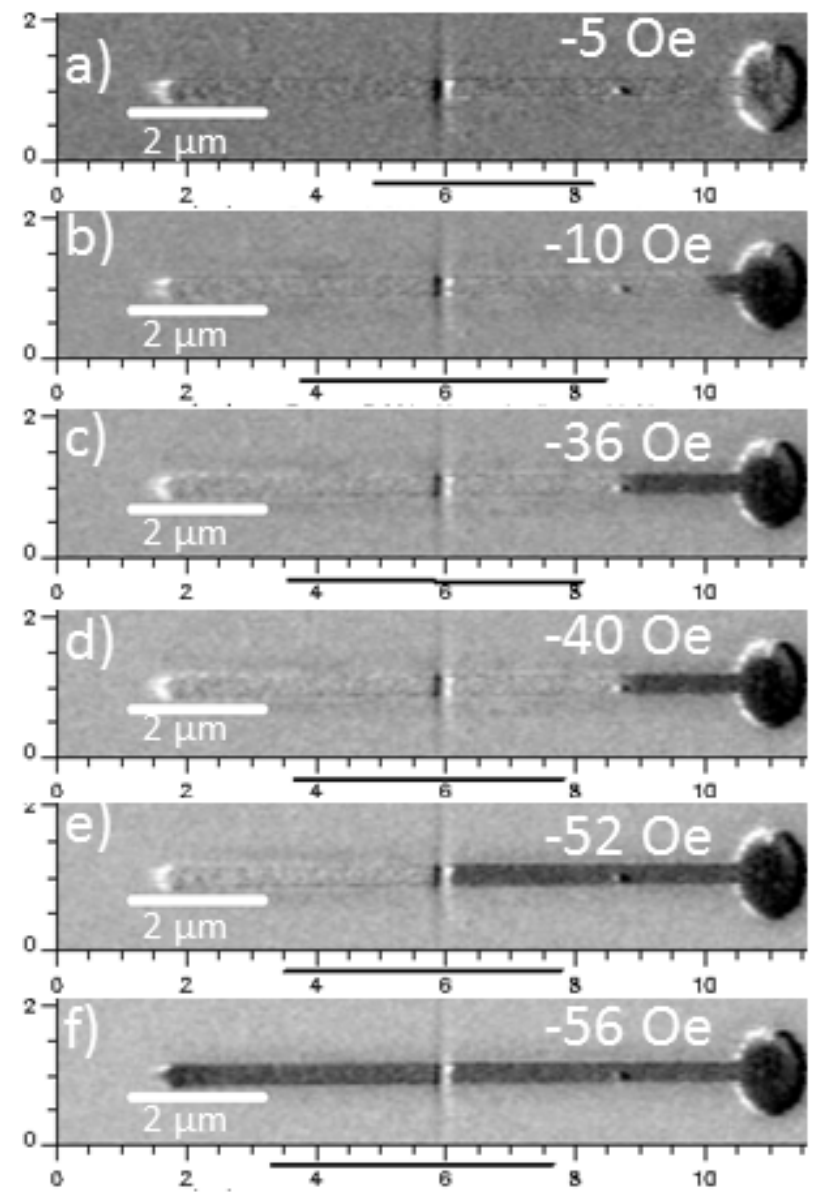